\title{
Determinants of diaphragm thickening fraction during mechanical ventilation: an ancillary study of a randomised trial
}

\author{
To the Editor:
}

Ultrasonography of the diaphragm is the subject of a growing interest in the intensive care unit (ICU) setting [1-6]. Observing the diaphragm in its zone of apposition allows measurement of its thickness and computation of its thickening fraction (TFdi), which depends on diaphragmatic activity [3] and reflects the diaphragm work of breathing [1]. A recent study showed that the TFdi correlated well with the endotracheal pressure variation generated by phrenic stimulation [6]. This index was also proposed for clinical evaluation of diaphragm weakness to detect ventilator-induced diaphragmatic dysfunction (VIDD) and predict difficult weaning [3, 4]. However, it remains unclear whether increased thickening in this setting only reflects a better intrinsic diaphragmatic strength, or if it also suggests enhanced work of breathing in response to increased cardiorespiratory workload. Furthermore, some authors suggested that VIDD could be thought as the "respiratory" manifestation of a global neuromuscular weakness [4, 7], but its relationship with ICU-acquired limb weakness is not straightforward [5]. The present study had a dual objective: first, to explore the correlation between ICU-acquired limb weakness (as assessed by the Medical Research Council (MRC) score) and diaphragm thickening (as assessed by TFdi); second, to assess the association of clinical variables with TFdi during mechanical ventilation.

This was a planned a priori ancillary study performed in one (Henri Mondor University Hospital, Creteil, France) of the nine participating centres of the B-type natriuretic peptide for the fluid Management of Weaning (BMW) trial [8]. We explored diaphragm thickening at the very beginning of weaning in 55 consecutive participants enrolled in this trial at this centre when ultrasonography was available. Ultrasonography was performed after $5 \mathrm{~min}$ of minimal respiratory support (pressure support set at $7 \mathrm{cmH}_{2} \mathrm{O}$ with zero end expiratory pressure), using an Envisor system (Philips Ultrasound, Bothell, WA, USA) equipped with a $12 \mathrm{MHz}$ high-resolution ultrasound linear probe. After locating the right hemi-diaphragm zone of apposition, the end-inspiratory and end-expiratory thicknesses were measured, allowing calculation of the TFdi of each patient, as previously reported [1]. The ultrasonography scans were performed by two intensivists, both experienced in ultrasonography (E. Vivier or F. Roche-Campo) and all measurements were analysed by E. Vivier. ICU-acquired weakness was screened in cooperating patients by clinical assessment of the limb strength, using the MRC score [9]. The most clinically relevant variables concerning diaphragmatic strength were used for the statistical analysis.

Spearman coefficients were computed to test pairwise correlations. These correlations were further used to build a focused principal component analysis (FPCA; "psy” package, R 3.2.2, the R Foundation for Statistical Computing, Vienna, Austria), using TFdi as the dependent variable and allowing a simple graphical display of correlation structures of clinical variables recorded before diaphragm ultrasonography [8].

We found no correlation between TFdi and MRC score (Spearman's rho correlation coefficient -0.07 ; $\mathrm{p}=0.62$ ) (figure 1a). FPCA using TFdi as outcome variable and variables collected prior to inclusion as independent variables revealed two main clusters and one isolated covariate (figure 1b): the first cluster included variables significantly positively correlated with TFdi: older age, cardiac and respiratory comorbidities (including chronic cor pulmonale, chronic obstructive pulmonary disease) and higher values of arterial carbon dioxide tension $\left(\mathrm{PaCO}_{2}\right)$ at inclusion; the second cluster included ICU treatments (need for neuromuscular blockade or vasopressor) and complications (ventilator-associated pneumonia and

@ERSpublications

Diaphragm thickening does not correlate with ICUAW; it is influenced by cardiopulmonary load and residual sedation http://ow.ly/6TC130e6G3Q

Cite this article as: Vivier E, Roche-Campo F, Brochard L, et al. Determinants of diaphragm thickening fraction during mechanical ventilation: an ancillary study of a randomised trial. Eur Respir J 2017; 50: 1700783 [https://doi.org/10.1183/13993003.00783-2017]. 


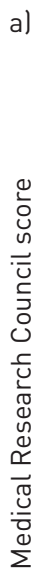

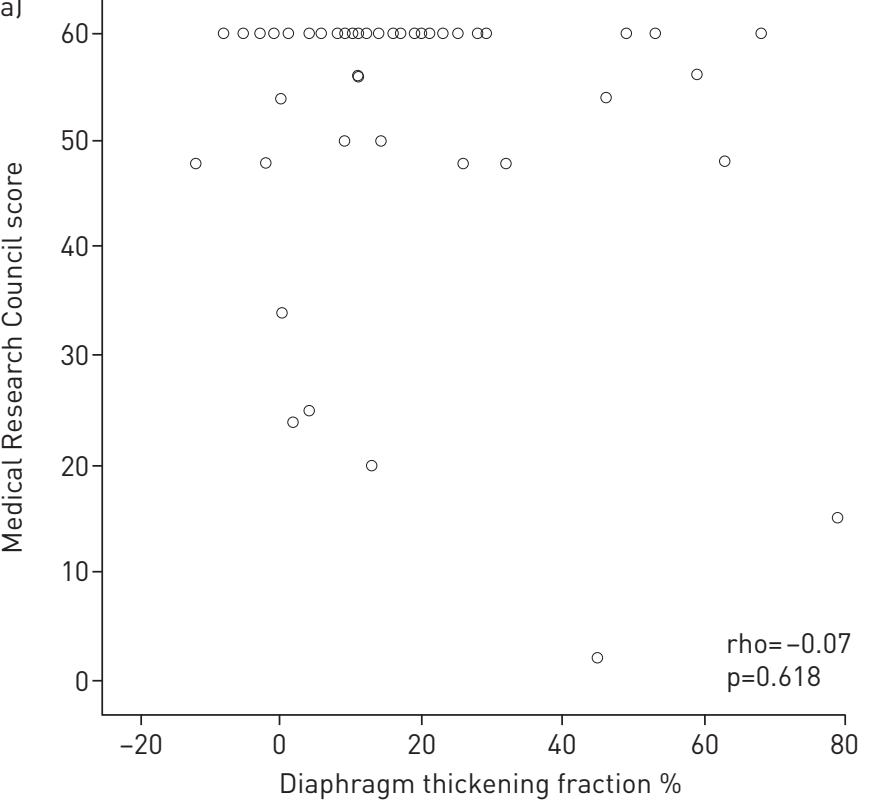

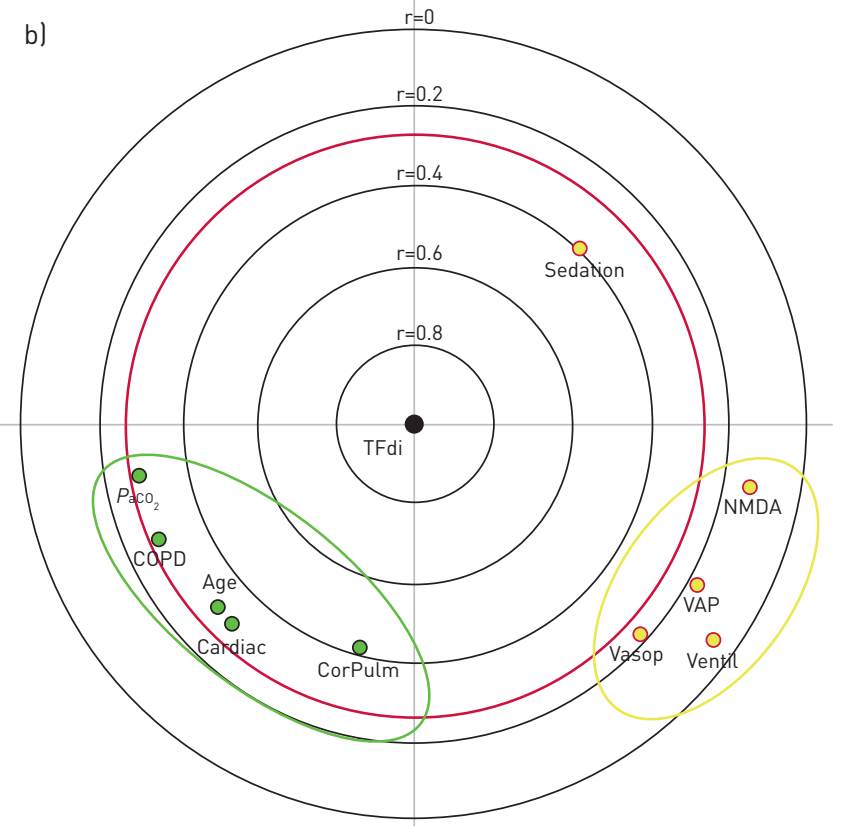

FIGURE 1 Correlation between Medical Research Council score and diaphragm thickening fraction in ventilated patients at initiation of weaning (a), and focused principal component analysis (FPCA) of diaphragmatic thickening fraction and patient characteristics (b). FPCA is a simple graphical display of correlation structures focusing on a particular dependent variable. The display reflects primarily the correlations between the dependent variable and all other variables (covariates) and secondarily the correlations among the covariates. The dependent variable (thickening fraction) is at the centre of the diagram and the distance of this point to a covariate faithfully represents their pairwise Spearman correlation coefficient lusing ranked values of continuous variables). Green covariates are positively correlated to the dependent variable, whereas yellow covariates are negatively correlated to the dependent variable. Covariates displayed inside the red circle are significantly correlated to the dependent variable $(p<0.05)$. The diagram also shows relationships between covariates as follows: correlated covariates are close (for positive correlations, allowing identification of clusters, as shown within the green or yellow ellipses) or diametrically opposite vis-à-vis the origin (for negative correlations) whereas independent covariates make a right angle with the origin. TFdi: diaphragmatic thickening fraction; Cardiac: any chronic cardiac disease; COPD: chronic obstructive pulmonary disease; CorPulm: chronic cor pulmonale; Age: age at inclusion; $P_{a c O_{2}}$ : arterial carbon dioxide tension at inclusion; Sedation: days not free from sedation before inclusion; NMDA: use of neuromuscular blockers before inclusion; VAP: ventilator-associated pneumonia before inclusion; Vasop: vasopressor use before inclusion; Ventil: days with ventilation before inclusion.

duration of ventilation) which had a negative but non-significant correlation with TFdi; last, the sedation burden (as assessed by the number of days not free from sedation) was significantly negatively correlated with TF.

It has been suggested that diaphragmatic dysfunction in patients receiving mechanical ventilation may be part of a global ICU-acquired weakness (ICU-AW) [7]. However, recent data seem to contradict this hypothesis [4, 5]. While DRES et al. [5] found a very weak correlation ( $\mathrm{rho}=0.28, \mathrm{p}=0.01)$ between MRC score and TFdi, we did not find any significant association between these variables. Although these two clinical assessment methods are different in nature (one is volitional and the other is objective), these results provide an argument in favour of an alternative impairment of the diaphragm.

The TFdi measured in the zone of apposition is inversely related to its shortening as the muscle volume remains constant [10]. This index reflects the work of breathing developed by the diaphragm in response to a given load; it can reflect intrinsic diaphragm strength (allowing diagnosis of diaphragm paresis or paralysis) but it is also influenced by the magnitude of the load imposed to the respiratory system, as shown during noninvasive or invasive assisted ventilation $[1,11]$. In our series, patients with a higher TFdi at initiation of weaning suffered more often from chronic cardiac or pulmonary disease and had a higher $\mathrm{PaCO}_{2}$ at weaning initiation than their counterparts. The thickening fraction was recorded with the same ventilator settings (minimal pressure support level) in all patients. A higher TFdi may be the expression of augmented work of breathing in response to an increased cardiorespiratory load imposed on the diaphragmatic muscle when assessed under spontaneous breathing conditions $[1,11]$. The thickening of the diaphragm in its zone of apposition may potentiate the diaphragmatic excursion when facing an increase in physiological (maximum inspiration) or pathological (dyspnoea) load in a well-awake patient. The significant negative correlation of TFdi with sedation suggests an influence of prolonged or residual sedation on respiratory drive and/or diaphragm function. Sedation exerts a well-known inhibitory effect on diaphragmatic contractility $[12,13]$. Diaphragmatic activity may be jeopardised by a residual sedation, 
especially in the early weaning period [14]. Last, TFdi was negatively but not significantly associated with early ICU treatments (neuromuscular blockers or vasopressor use before inclusion) and complications (ventilator-associated pneumonia and prolonged ventilation), which may be risk factors for diaphragmatic atrophy and weakness [15]. The lack of significance of these associations may be due at least in part to the small size of our cohort.

The strengths of our study include the standardisation of ventilator setting during diaphragm ultrasonography and the early assessment of TFdi at the initiation of weaning. However, two limitations must be considered: first, we did not compare the diaphragm thickening fraction to a clinical indicator of inspiratory force (such as maximal inspiratory pressure at the airways); second, the low interobserver reproducibility of TFdi values $[1,3]$ requires caution when interpreting our data in the clinical scenario.

In conclusion, our data corroborate recent studies reporting no tight association between diaphragm activity and limb weakness in critically ill patients. We observed a positive correlation between cardiopulmonary comorbidities and TFdi suggesting a possible influence of respiratory load on TFdi when assessed under minimal respiratory support. Conversely, a negative correlation between exposure to sedation and TFdi suggested an impairment of diaphragm activity by residual sedation.

Emmanuel Vivier ${ }^{1,2}$, Ferran Roche-Campo ${ }^{3,4}$, Laurent Brochard ${ }^{5,6}$ and Armand Mekontso Dessap ${ }^{2,4}$

${ }^{1}$ Hôpital Saint Joseph Saint Luc, Service de Réanimation Polyvalente, Lyon, France. ${ }^{2}$ Université Paris Est Créteil, Faculté de Médecine de Créteil, IMRB, Groupe de recherche clinique CARMAS, Créteil, France. ${ }^{3}$ Hospital Verge de la Cinta, Servei de Medicina Intensiva, Tortosa, Spain. ${ }^{4}$ AP-HP, Hôpitaux Universitaires Henri Mondor, DHU A-TVB, Service de Réanimation Médicale, Créteil, France. ${ }^{5}$ Interdepartmental Division of Critical Care Medicine, University of Toronto, Toronto, ON, Canada. ${ }^{6}$ Keenan Research Centre and Li Ka Shing Knowledge Institute, St Michael's Hospital, Toronto, ON, Canada.

Correspondence: Emmanuel Vivier, Service de Réanimation Polyvalente, Centre Hospitalier Saint Joseph Saint Luc, 20 Quai Claude Bernard, 69007 Lyon, France. E-mail: evivier@ch-stjoseph-stluc-lyon.fr

Received: Aug 292016 | Accepted after revision: June 192017

Conflict of interest: Disclosures can be found alongside this article at erj.ersjournals.com

\section{References}

1 Vivier E, Mekontso Dessap A, Dimassi S, et al. Diaphragm ultrasonography to estimate the work of breathing during non-invasive ventilation. Intensive Care Med 2012; 38: 796-803.

2 DiNino E, Gartman EJ, Sethi JM, et al. Diaphragm ultrasound as a predictor of successful extubation from mechanical ventilation. Thorax 2014; 69: 423-427.

3 Goligher EC, Fan E, Herridge MS, et al. Evolution of diaphragm thickness during mechanical ventilation. Impact of inspiratory effort. Am J Respir Crit Care Med 2015; 192: 1080-1088.

4 Jung B, Moury PH, Mahul M, et al. Diaphragmatic dysfunction in patients with ICU-acquired weakness and its impact on extubation failure. Intensive Care Med 2016; 42: 853-861.

5 Dres M, Dubé B-P, Mayaux J, et al. Coexistence and impact of limb muscle and diaphragm weakness at time of liberation from mechanical ventilation in medical intensive care unit patients. Am J Respir Crit Care Med 2017; 195: 57-66.

6 Dubé B-P, Dres M, Mayaux J, et al. Ultrasound evaluation of diaphragm function in mechanically ventilated patients: comparison to phrenic stimulation and prognostic implications. Thorax 2017; in press [https://doi.org/10. 1136/thoraxjnl-2016-209459].

7 De Jonghe B, Bastuji-Garin S, Sharshar T, et al. Does ICU-acquired paresis lengthen weaning from mechanical ventilation? Intensive Care Med 2004; 30: 1117-1121.

8 Mekontso Dessap A, Roche-Campo F, Kouatchet A, et al. Natriuretic peptide-driven fluid management during ventilator weaning: a randomized controlled trial. Am J Respir Crit Care Med 2012; 186: 1256-1263.

9 Kleyweg RP, van der Meché FG, Schmitz PI. Interobserver agreement in the assessment of muscle strength and functional abilities in Guillain-Barré syndrome. Muscle Nerve 1991; 14: 1103-1109.

10 Ueki J, De Bruin PF, Pride NB. In vivo assessment of diaphragm contraction by ultrasound in normal subjects. Thorax 1995; 50: 1157-1161.

11 Umbrello M, Formenti P, Longhi D, et al. Diaphragm ultrasound as indicator of respiratory effort in critically ill patients undergoing assisted mechanical ventilation: a pilot clinical study. Crit Care Lond Engl 2015; 19: 161.

12 Molliex S, Dureuil B, Montravers P, et al. Effects of midazolam on respiratory muscles in humans. Anesth Analg 1993; 77: 592-597.

13 Zhang X-J, Yu G, Wen X-H, et al. Effect of propofol on twitch diaphragmatic pressure evoked by cervical magnetic stimulation in patients. Br J Anaesth 2009; 102: 61-64.

14 Rozé H, Germain A, Perrier V, et al. Effect of flumazenil on diaphragm electrical activation during weaning from mechanical ventilation after acute respiratory distress syndrome. Br J Anaesth 2015; 114: 269-275.

15 Berger D, Bloechlinger S, von Haehling S, et al. Dysfunction of respiratory muscles in critically ill patients on the intensive care unit. J Cachexia Sarcopenia Muscle 2016; 7: 403-412. 